\title{
Effects of the Cranio-cervical Static Stabilization Exercises among the Using Small Tools
}

\author{
Won-Sik Bae, MS, PT • Keon-Cheol Lee, PhD, $\mathrm{PT}^{\dagger} \cdot$ Hyun-Ok Lee, PhD, $\mathrm{PT}^{1}$ \\ Department. of Physical Therapy, Kyungnam College of Information \& Technology \\ ${ }^{1}$ Department. of Physical Therapy, Catholic University of Pusan
}

Received: June 23, 2016 / Revised: July 4, 2016 / Accepted: August 10, 2016

(c) 2016 J Korean Soc Phys Med

\section{| Abstract |}

PURPOSE: The purpose of this study is to determine the differences between the muscle activity of cranio-cervical flexion and extension muscles according to the types of tools used through a short-term intervention of cranio-cervical static stabilization exercises using small tools.

METHODS: A total of fifteen male and female adults in their 20s who showed forward head posture in the overall body posture measurement system participated in this study. Each subject performed cranio-cervical static stabilization exercises about flexion and extension while using a sling, a foam roller, a TOGU ball, and without tools separately, and the muscle activity of the sternocleidomastoid muscle, scalenus anterior and splenius capitis was measured. Each value was measured for 10 seconds a total of three times. The maximum voluntary isometric contraction value was computed using the average during the middle four seconds.

RESULTS: Cranio-cervical flexion exercises using various tool types, the average activity of the sternocleidomastoid and

†Corresponding Author : rptgeon@naver.com

This is an Open Access article distributed under the terms of the Creative Commons Attribution Non-Commercial License (http://creativecommons.org/licenses/by-nc/3.0) which permits unrestricted non-commercial use, distribution, and reproduction in any medium, provided the original work is properly cited. scalenus anterior muscles was significantly higher when applying the TOGU ball $(\mathrm{p}<.05)$. According to the results of implementing cranio-cervical flexion exercise using various tools, the maximum muscle activity of the sternocleidomastoid muscle was significantly higher for the TOGU ball $(\mathrm{p}<.05)$.

CONCLUSION: Based on these results, the provision of an unstable surface using small tools rather than a stable surface is recommended as an exercise scheme for proprioceptive stimulation in a forward head posture. Particularly, we recommend using the TOGU ball for the provision of an unstable surface to increase the muscle activity of the sternocleidomastoid muscle and scalenus anterior.

Key Words: EMG, Foam roller, Forward head posture, Head-neck muscle, TOGU

\section{Introduction}

Forward head posture generates mechanical stress of the neck and head by structurally moving the central line of the head forward and upward (Waris, 1979). As the weight of the head that the neck supports increases, relative 
compensation is incurred, including increased extension in the linkage between the skull and the neck, abnormal and continuous muscle contraction of the muscles under the back of the head, the neck muscles, and shoulder muscles. This induces change in the linkage between the head and neck (Harrison et al., 2003) and hence is pointed out as an important cause of musculoskeletal pain symptoms (Cailliet, 1998).

Small tools that provide unstable surfaces are frequently used in improving proprioceptive sensations (Creager, 1996). Recently, exercise therapy schemes that use small tools for the neck have received increasing attention; a sling, a TOGU, a gym ball, a foam roller, and a biofeedback stabilizer are mainly used. These somesthesia-motor training tools are proven to be effective in improving posture control (Lim, 2012). Since these exercises are performed on a trembling surface, they awaken the brain by activating the proprioceptive receptors to quicken information processing speed, which improves physical agility and power as well as coordination power. Somesthesia-motor control means the controllability of the central nervous system on motion, balancing, posture, and joint stability (Franklin and Wolpert, 2011). The muscle spindles (Banks, 2006) in all skeletal muscles are the most important origin of proprioception (Proske and Gandevia, 2012), and they are diversely distributed in body. In particular, the muscles of the neck under the back of the head have high muscle spindle density due to the role of neck vertebra in head-eye movement control (Liu et al., 2003). Agility, joint stability, coordination, and balancing during neck movement are controlled by the proprioceptive system (Milner et al., 2007).

In another study, a regular sling bridge exercise was found to reduce the pain of patients with back pain and increase overall muscle activity more than other tools (Jeong et al., 2013). The findings of another study indicated that abdominal muscle exercise using a sling and a Swiss ball on an unstable surface increased abdominal muscle activity (Lee and Kim, 2014), and in a study that examined the muscle activity of the body trunk according to posture change in a bridge exercise with a gym ball reported a significant difference (Son et al., 2009). Moreover, it was proven that using a gym ball as an exercise tool to strengthen core muscles can increase trunk joint flexibility and muscle strength, as it develops spinal stability and flexibility (Jespersen and Potvin, 2000). Moreover, Jung et al. (2010) reported that a one-foot holding position exercise using a foam roller increased the thickness of the deep abdominal muscles more than on a stable surface, as the muscle activity increased.

However, comparison studies examining the characteristics of small tools that provide unstable surfaces are scarce. Hence, this study attempts to compare the differences in the muscle activity of cranio-cervical flexion and extension muscles according to the specific tools used in a short-term intervention of cranio-cervical static stabilization exercises using small tools.

\section{Method}

\section{Study subjects}

A total of 15 male and female college students in their 20s who were enrolled in University K located in Busan Metropolitan City, Korea were selected as study subjects. They had no neurologic or musculoskeletal disease history or functional defects that could affect the study results. Those who were not doing regular or systematic exercise at the time of subject selection and those who showed forward head posture over $2.5 \mathrm{~cm}$ in the overall body posture measurement system were chosen as the subjects (Salahzadeh et al., 2014). All the subjects were provided with a sufficient explanation about the study content and participated in the study after signing a consent form. 


\section{Measurement tools and method}

1) Overall body posture measurement system

In this study, the overall body posture measurement system (GPS400, Chinesport, Italy) was used as an experimental device for measuring forward head posture (Fig. 1). Images are captured from a fixed location $3 \mathrm{~m}$ away for the posture measurement. A sticker was attached to the acromion, and the distance between the central line of the meatus acusticus externus and a vertical line with the acromion as an axis was measured (Lee, 2011).

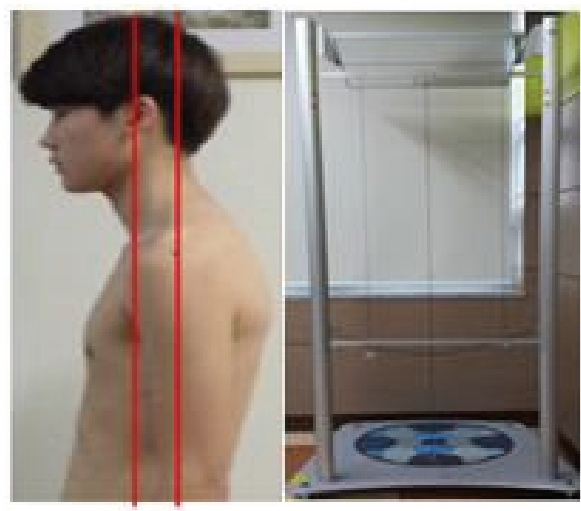

Fig. 1. Forward head posture measurement

\section{2) Electromyogram measurement tool}

A surface electromyogram device (Telemyo - DTS, NORAXON, USA) was used to measure the muscle activity of the sternocleidomastoid muscle, scalenus and splenius capitis of the study subjects (Fig. 2). Electrodes were attached after wiping alien substances off the skin using alcohol cotton to minimize the development of resistance created in the skin against the electromyogram signal. Record electrodes were attached to the left side of the sternocleidomastoid muscle and splenius capitis and the right scalenus. The sample extraction rate of the electromyogram signal was set at $1024 \mathrm{~Hz}$. As for the frequency bandwidth, a frequency band-pass filter of the Bagnoli EMG system of $10350 \mathrm{~Hz}$ and a notch filter of $60 \mathrm{~Hz}$ was used. The root mean square (RMS) was analyzed following full-wave rectification of the collected muscle activity signal during cranio-cervical flexion and extension using the Acquisition and Analysis Software (Delsys, U.S.A.) program, and it was quantified to maximal voluntary isometric contraction (MVIC).

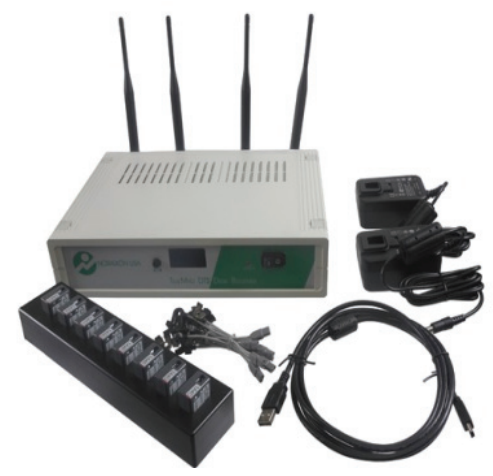

Fig. 2. Electromyogram measurement tool

3) Measurement method

(1) Scalenus anterior and sternocleidomastoid muscle

For the scalenus anterior, an electrode was attached to the triangular point formed by the back of the sternocleidomastoid muscle, above the clavicle and the upper trapezius. For the sternocleidomastoid muscle, an electrode was attached to the middle point of the mastoid and the upper notch in the sternum (O'Leary et al., 2011). The subjects were in a prone position, and the tool was placed near their forehead. With Halvorsen (2010) method, subjects contracted for 10 seconds isometrically with chin-tuck (Fig. 3). In a repeated measurement three times,

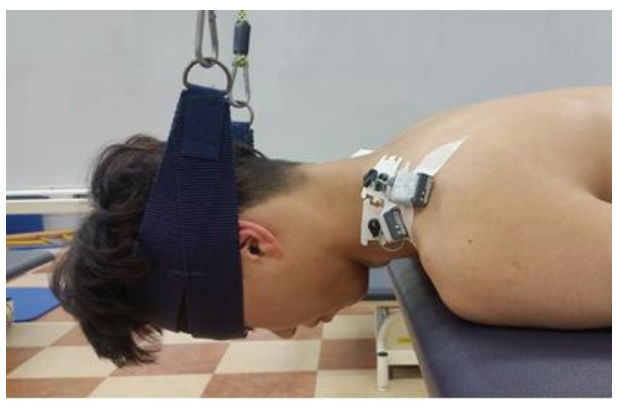

Fig. 3. Measurement position 
a resting time of 10 second was applied between each measurement to minimize fatigue (Yun and Kim, 2013) and the MVIC was computed using the average during the four seconds in the middle.

\section{(2) Splenius capitis}

For the splenius capitis, a surface electrode was attached to the center between the sternocleidomastoid muscle and upper trapezius at the second and third neck bone height (Falla et al., 2008). The subjects were in a supine position, and the tool was placed near their occipital bone. With Halvorsen (2010) method, subjects contracted for 10 seconds isometrically with chin-tuck. In a repeated measurement three times, a resting time of 10 second was applied between each measurement to minimize fatigue (Yun and Kim, 2013), and MVIC was computed using the average during the four seconds in the middle.

\section{Exercise tools}

1) Sling

A sling (Sling, Green TESMA, Korea) is an exercise tool using the movement of a hanging point, control of the length of a sling string and the principle of levers. It is possible to change the treatment intensity by altering the length of sling-mechanical joint, the manual resistance of the therapist, the applied weight, and the use of an elastic band (Chae, 2002).

\section{2) Foam roller}

A foam roller (Tratac foam roller, XTRATAC, Korea) is a small, lightweight tool that is made of rubber. It has excellent impact-absorbing power or a high-quality sponge (made with EVA). It generally has a cylindrical shape in a long circular stick or semicircular shape.

3) TOGU ball

A TOGU ball is a ball injected with air (TOGU, DYN Air Ballkissen, Germany) that is used as a tool for sensory exercise that activates muscles that are generally not used during daily activity. This sensory training control activates small muscles instead of large muscles and enhances energy consumption.

\section{Analysis method}

The SPSS ver. 22.0 program for Windows (SPSS, Chicago, IL, USA) was used for the statistical analysis of the data. One-way ANOVA was used to compare the differences between the muscle activity of cranio-cervical flexion and extension muscles. The statistical significance level was set at $a=.05$.

\section{Results}

1. General characteristics of the research subjects

The general characteristics of the research subjects are summarized in Table 1. A total of 15 subjects participated in this study, whose average age was $21.67 \pm 1.55$, height was $167.2 \pm 7.43 \mathrm{~cm}$, and weight was $62.29 \pm 9.58 \mathrm{~kg}$.

2. Muscle activity in the cranio-cervical flexion exercise

In the cranio-cervical flexion exercise, the average muscle activity of the sternocleidomastoid muscle and scalenus anterior was significantly higher when applying

Table 1. General characteristics of subjects

\begin{tabular}{cccc}
\hline \multirow{2}{*}{ Sex (Male/Female) $(\mathrm{n}=15)$} & Age (years) & Body weight $(\mathrm{kg})$ & Height $(\mathrm{cm})$ \\
\cline { 2 - 4 } & $\mathrm{M} \pm \mathrm{SD}$ & $\mathrm{M} \pm \mathrm{SD}$ & $\mathrm{M} \pm \mathrm{SD}$ \\
\hline $8 / 7$ & $21.67 \pm 1.55$ & $62.29 \pm 9.58$ & $167.2 \pm 7.43$ \\
\hline
\end{tabular}


Table 2. Comparison of the mean EMG activity among the using device in the anterior muscle

(unit: \%MVIC)

\begin{tabular}{ccccccc}
\hline Muscle & Sling & Foam roller & TOGU & No device & F & p \\
\hline SCM & $170.21 \pm 80.68$ & $189.50 \pm 95.38$ & $220.59 \pm 103.71$ & $126.53 \pm 60.17$ & 3.10 & $.03^{*}$ \\
Ant. Scalenus & $115.96 \pm 51.63$ & $129.42 \pm 54.32$ & $148.03 \pm 62.82$ & $93.60 \pm 39.59$ & 2.83 & $.04^{*}$ \\
\hline
\end{tabular}

Table 3. Comparison of the peak EMG activity among the using device in the anterior Muscle

(unit: \%MVIC)

\begin{tabular}{ccccccc}
\hline Muscle & Sling & Foam roller & TOGU & No device & F & p \\
\hline SCM & $236.86 \pm 115.32$ & $269.97 \pm 133.09$ & $301.11 \pm 140.99$ & $175.09 \pm 85.52$ & 3.00 & $.04^{*}$ \\
Ant. Scalenus & $171.26 \pm 77.11$ & $180.40 \pm 80.63$ & $214.42 \pm 96.16$ & $150.43 \pm 75.21$ & 1.56 & .21 \\
\hline
\end{tabular}

the TOGU ball $(\mathrm{p}<.05)$. According to the post-hoc test results based on LSD test method, the TOGU showed a significant difference compared to the use of no tool $(p<.05)$ (Table 2).

The maximum muscle activity of the sternocleidomastoid muscle in the cranio-cervical flexion exercise was significantly higher when the TOGU ball was used $(p<.05)$. According to the post-hoc test results based on LSD test method, the TOGU ball and foam roller showed significant differences compared to the use of no tool $(\mathrm{p}<.05)$. The maximum muscle activity of the scalenus anterior was the highest for the TOGU ball, but this was not significant (Table 3).

\section{Muscle activity in the cranio-cervical extension} exercise

In the cranio-cervical extension exercise, the average and maximum muscle activities of the splenius were highest for the TOGU ball, but this was not significant.

\section{Discussion}

This study applied a sling, foam roller, and TOGU ball to adults in their 20 s who have forward head posture and compared the differences in their neck muscle activity during cranio-cervical static stabilization exercises.
The results indicated the highest average and maximum muscle activities of the sternocleidomastoid muscle and scalenus anterior when a TOGU ball was applied during the cranio-cervical flexion exercise. Moreover, the splenius capitis also showed the highest muscle activity with the use of the TOGU ball during the extension exercise, despite no statistical significance. In terms of previous studies, $\mathrm{Oh}$ (2012) applied a TOGU exercise program to 20 elderly people and reported that standing position TOGU exercises improved their balancing ability. Kim and Lee (2012) reported that subjects' overall body balance ability enhanced following a TOGU exercise regimen. Based on these results, it is surmised that TOGU exercise induces lateral flexion and rotation of the head and neck to stimulate proprioception in the neck, which consequently improves balance and agility. It is conjectured that providing a more unstable surface than a foam roller and sling resulted in the stimulation of proprioception, inducing larger muscle activity.

In cranio-cervical flexion exercise, the maximum muscle activity of the sternocleidomastoid muscle was also significantly higher in the case of using a foam roller compared to no tool. A foam roller, which is a small tool for rehabilitation exercise, is being used on unstable surfaces to strengthen balancing reactions against rotational movement on a horizontal surface (Jung et al., 2010). Moreover, foam rollers can also be used for the purpose 
of self-myofascial release exercise (Kim et al., 2011), improvements in balancing ability, and the strengthening of deep muscles aimed at rehabilitation treatment or posture correction (Bae and Baek, 2015). McMeeken et al. (2004) argued that thickness changes are highly correlated with muscle activity changes in the transverse abdominis. Jung et al. (2010) reported that muscle activity increased when applying a single-leg holding exercise using a foam roller, as the thickness of the abdominal muscle increased more, compared to the case of using a stable surface. Hence, we can say that exercise on a foam roller increased muscle activity more than on a stable surface.

In the present study, a sling showed higher muscle activity compared to no tool in both the cranio-cervical flexion and extension exercises, despite no statistical significance. It is reported that sling exercises have therapeutic effects on mobility, extension, somesthesiamotor training, muscle stabilization, muscle strengthening, muscle endurance improvement, and release (Kim and Kwon, 2001). A sling induces lateral flexion of the head and neck during cranio-cervical flexion and extension exercises. It supports the head comfortably and stably and the diverse neck exercises can be provided by controlling the sling height (Chae, 2002). As a result, it is surmised that sling exercises also induced larger muscle activity than a stable surface by stimulating the proprioceptive system around the neck. Kim et al. (2011) applied a sling exercise program to adults in their 20s who have forward head posture as an exercise for strengthening their head flexor muscle and reported that the experimental group showed significantly increased muscle activity of the serratus anterior and lower trapezius compared to the control group. Yun and Kim (2013) applied a cranio-cervical flexion exercise using a sling to patients with chronic neck pain and reported that the thickness of the sternocleidomastoid muscle increased and that it was effective for patients who have a hyper-activated sternocleidomastoid muscle.

This study has several limitations. First, the study subjects included adults in their 20s only and the sample size was small, making the results difficult to generalize. Moreover, impacts from neighboring muscles were not controlled when measuring the muscle activity, and hence it was hard to obtain pure muscle activity signals. Hence, it will be necessary to increase the usability of TOGU exercise in the future through additional studies that consider more diverse age groups and larger numbers of subjects and that can obtain pure muscle activity signals.

\section{Conclusion}

The sternocleidomastoid muscle and scalenus anterior showed the highest average and maximum muscle activity when using the TOGU ball during cranio-cervical static stabilization exercise. The maximum muscle activity of the sternocleidomastoid muscle was significantly higher when applying a foam roller than without a tool. Moreover, the splenius capitis showed the highest muscle activity when applying the TOGU ball during static stabilization exercise, despite no statistical significance. The muscle activity was higher for the sling application than with no tool, but there was no statistical significance. Based on these results, the provision of an unstable surface using small tools rather than a stable surface is recommended as an exercise scheme for proprioceptive stimulation in a forward head posture. In particular, we recommend using the TOGU ball for the provision of an unstable surface to increase the muscle activity of the sternocleidomastoid muscle and scalenus anterior.

\section{References}

Bae YH, Baek HY. Safe delivery pilates using props. Seoul. Morning Scene. 2015.

Banks RW. An allometric analysis of the number of muscle 
spindles in mammalian skeletal muscles. J Anat. 2006;208(6):753-68.

Chae YW. The measurement of forward head posture and pressure pain threshold in neck muscle. J Korean Soc Phys Ther. 2002;14(1):117-24.

Cailliet R. Soft tissue pain and disability. ( $2^{\text {nd }}$ ed). Philadelphia. FA Davis. 1998.

Creager CC. Therapeutic exercises using foam rollers. Berthoud. Executive Physical Therapy, Inc. 1996.

Falla D, Farina D, Kanstrup DM, et al. Pain-induced changes in cervical muscle activation do not affect muscle fatigability during sustained isometric contraction. J Electromyogr Kinegiol. 2008;18(6):938-46.

Franklin DW, Wolpert DM. Computational mechanisms of sensorimotor control. Neuron. 2011;72(3):425-42.

Halvorsen FH. Neuracl seminer workbook. RedcordAS. prone cervical setting test procedure 91. 2010;3:5-7.

Harrison DE, Harrisson DD, Bets JT. Increasing the cervical lordosis with chiropractic biophysics seated combined extension compression and transverse load cervical traction with cervical manipulation: Nonrandomized clinical control trial. J Manipulative Physiol Ther. 2003;26(3):139-51.

Jeong ED, Woo C, Chaea HKY, et al. The effects of sling bridging exercise to pain scale and trunk muscle activity in low back pain patients. J Int Acad Phys Ther Res. 2013;4(1):479-544.

Jespersen M, Potvin AN. The great body ball handbook. Productive fitness products, Inc. 2000.

Jung DY, Koh EK, Kim SJ, et al. Comparisons of abdominal muscles thickness during single leg holding exercise on stable surface and on a foam roller using ultrasound imaging. Korean J Sport Biomech. 2010;20(4): 415-20.

Kim EJ, Kim JW, Park BR. Effects of sling exercise program on muscle activity and cervical spine curvature of forward head posture. Korea Contents Soc. 2011; 11(11):213-20.
Kim HJ, Lee AL, Yun MJ, et al. Pilates complete conquest using props. Seoul. Morning Scene. 2011.

Kim SJ, Lee KJ. The effect of TOGU exercise on the postural balance and foot pressure distribution. J Korean Acad Phys Ther. 2012;19(1):9-15.

Kim SY, Kwon JH. Lumbar stabilization exercises using the sling system. J Korean Acad Orthop Man Ther. 2001;7(2):23-39.

Lee DH. The effects of balance exercise and stretching exercise on forward head posture. Doctor's Degree. Daegu University. 2011.

Lee MH, Kim BK. Comparison of abdominal muscle activity after sling and swiss-ball exercises in asymptomatic adults. J Korean Soc Phys Med. 2014;9(3):333-8.

Lim CH. Comparision of the muscle activity and balance of lower extremities in exercise using TOGU on the unstable surface and stable surface after reconstruction of the ACL. J Korean Soc Phys Med. 2012;7(3):251-8.

Liu J, Thornell L, Pedrosa-Domellof F. Muscle spindles in the deep muscles of the human neck: a morphological and immunocytochemical study. J Histochem Cytochem. 2003;51(2):175-86.

McMeeken JM, Beith ID, Newham DJ, et al. The relationship between EMG and change in thickness of transversus abdominis. Clin Biomech. 2004;19(4):337-42.

Milner TE, Hinder MR, Franklin DW. How is somatosensory information used to adapt to changes in the mechanical environment? Comput Neurosci Thero Insights Brain Funct. 2007;165:363-72.

Oh SH. Comparing the effects between swiss ball and aero step exercise program on balance of standing posture of elderly. Master's Degree. Dankook University. 2012

O'Leary S, Falla D, Jull G. The relationship between superficial muscle activity during the cranio-cervical flexion test and clinical feature in patients with chronic neck pain. Man Ther. 2011;16(5):452-5.

Proske U, Gandevia SC. The proprioceptive senses: Their roles 
in signaling body shape, body position and movement, and muscle force. Physiol Rev. 2012;92(4):1651-97. Salahzadeh Z, Maroufi N, Ahmadi A, et al. Assessment of forward head posture in females: observational and photogrammetry methods. J Back Musculoskelet Rehabil. 2014;27(2):131-9.

Son ST, Kim MH, Kim HJ, et al. The comparison of trunk muscles activity during bridging stabilization exercises on swiss ball according to change of position. J Korean Soc Phys Med. 2009;4(4):221-9.

Waris P. Occupational cervicobrachial syndromes: a review. Scand J Work Environ Health. 1979;5(suppl3):3-14. Yun KH, Kim K. Effect of craniocervical flexion exercise using sling on thickness of sternocleidomastoid muscle and deep cervical flexor muscle. J Korean Soc Phys Med. 2013;8(2):253-61. 